\title{
Temperature induced dimensional tuning and anomalous deformation of micro/nanopores
}

Shusong Zhang, ${ }^{1 \neq}$ Zhenming Ji, ${ }^{1 \neq}$ Guanghua Du, ${ }^{2}$ Jie Liu, ${ }^{2}$ Xi Zhou,,$^{1 *}$ and Yanbo

$X i e^{1 *}$

${ }^{1}$ MOE Key Laboratory of Material Physics and Chemistry under Extraordinary

Conditions, Northwestern Polytechnical University, Xi'an 710072, China

2Institute of Modern Physics, Chinese Academy of Sciences, Lanzhou 730000, China.

*Corresponding author: zhouxi@nwpu.edu.cn; ybxie@nwpu.edu.cn 


\section{Methods}

Irradiation. The prepared PET was irradiated by Ta ion beams at room temperature with an energy of $12.5 \mathrm{MeV} / \mathrm{u}$ by accelerator SFC and SSC in Lanzhou for a single ion track and multiple-tracks (track density of $10^{5} \sim 10^{7}$ ) respectively. The single track on PET was achieved by placing a mask (100 $\mu \mathrm{m}$ Si wafer) in front of a microbeam. In order to obtain a nanopore of a specific density, the beam is immediately turned off when the detector detects the density of the corresponding flow.

Multipore membranes. The foils were UV irradiated before chemical etching, for at least 1.5 hours on each side. We immersed the foil in a $9 \mathrm{M} \mathrm{NaOH}$ bath for a certain time to obtain a symmetric structure of the nanopore. In order to prevent the film from floating on the etching solution, we clamped the film with a plastic clip to keep it completely immersed in the solution. The specimens were taken out of etchant and immersed in the stopping medium $(1 \mathrm{M} \mathrm{HCOOH}$ and $1 \mathrm{M} \mathrm{KCl})$ immediately after the etching process to make sure the chemical etching was stopped in time. Finally, the PET film with the pores was cleaned with DI water and stored in a dry environment.

Single track nanopore etching. Like for the multipore membranes we performed UV irradiation on the PET foil. We added $9 \mathrm{M}$ sodium hydroxide to both sides of the reservoir, and performed the electrical conductance measurement by $1 \mathrm{~V}$ to 
monitor the change of the pore size. We stopped the etching of the PET nanopore when the conduction increased to 100 nA. Finally, we added stopping medium to every side of the tank to stop etching for over 30 minutes.

Thermal treatment. We divided the etched membranes into pieces for the thermal treatment, ensuring that the pores were etched in the same conditions. The etched films were placed in a water bath from $50^{\circ} \mathrm{C}$ to $90^{\circ} \mathrm{C}$ with a step of $10^{\circ} \mathrm{C}$, where the specimens could be homogeneously heated. The water contained in a beaker was heated by an oil bath to remain at a stable temperature, characterized by a thermometer. We immersed the membrane into the water bath until the membrane showed no clear deformation any more, taking the results in Figure S1b as a reference.

\section{Calculations}

Shrinking rate. To quantitatively discuss the oriented shrinkage of the polymer foil, we defined a shrinking rate $\left(s_{i}\right)$ in the stretched direction (SD) and unstretched direction (UD) as follows,

$$
s_{i}=\frac{l_{0, i}-l_{s, i}}{l_{0, i}},
$$

where $l_{0, i}, l_{s, i}$ are the length before and after shrinkage in $i$ (SD and UD) respectively. We calculate the shrinking rate according to this definition, including the deformation of nanopores in the following sections. 
Residual stress. Previous studies ${ }^{1}$ showed that the residual stress of a polymer foil can be characterized by a change of refractive index $\Delta n$, measured by optical birefringence,

$$
\frac{\Delta n}{\sigma_{\text {bulk }}}=\frac{2 \pi\left(n^{2}+2\right)^{2}}{45 \quad n k T}\left(\alpha_{1}-\alpha_{2}\right)
$$

where $n$ and $\Delta n$ are the mean refractive index $(1.58 \text { for PET })^{1}$ and refractive index change after thermal shrinkage, $\alpha_{1}-\alpha_{2}$ is the polarizability of the random link $(0.7 \times 1$ $\left.0^{-23} \mathrm{~cm}^{3}\right)^{2}$, and $k$ and $T$ the Boltzmann constant and temperature. As we found $\Delta n=0.0248$ for the original film at $23^{\circ} \mathrm{C}$ in our measurements by an Abbe refractometer, the residual stress $\sigma_{\text {bulk }}$ of the polymer can be derived as $8.1 \mathrm{Mpa}$.

Equivalent nanopore radius of electrical conductance. Since the conical angle of the nanopore is less than 5 degrees, we can approximate the resistance by using a cylindrical model. We then derive the equivalent radius of the nanopore $r_{e q}$ from the resistance by

$$
r_{e q}=\sqrt{\frac{L}{\kappa \pi R}},
$$

where $L, \kappa$, and $R$ are the thickness of the deformed foil (equal to the length of nanopore), the conductivity of the solution $\left(10.79 \mathrm{~S} / \mathrm{m}\right.$ for $1 \mathrm{M} \mathrm{KCl}$ solution at $\left.23{ }^{\circ} \mathrm{C}\right)$ and the resistance from linear fitting of the I-V curve, respectively.

Here we neglected the resistance contribution from the complex deformation at the nanopore exterior surface, as the resistance is proportional to the length while the length of the exterior surface is negligible compared to the thickness of the entire deformed membrane $(40 \sim 110 \mu \mathrm{m})$. The equivalent nanopore radius $r_{e q}$ thus calculated is shown as the red solid dots in Figure 4c.

Prediction of the electrical resistance. We use the shrinkage data of polymer foil to predict the change of resistance of single nanopore. We fitted the data including the length of SD and thickness of foil at 23, 60, 70, 80 and 90 degrees in Figure S1c. The fitted SD length is expressed as $l_{S D}=-0.01867(T-23)+2 \mathrm{~cm}$. The fitting formula of thickness is $L=0.01618(T-23)^{2}+36.27 \mu m$, where $T$ is the temperature. For the almost constant 
length MD, we derive the average $l_{U D}=1.897 \mathrm{~cm}$ for prediction. Thus, the trend of the electrical resistance of single nanopore (solid green line in Figure 4c) can be predicted by the deformation of bulk polymer as the following equation

$$
R^{\prime}=\frac{4 L}{\kappa \pi l_{S D} l_{U D}}
$$

by setting $R^{\prime}$ at 23 degrees equal to the fitting resistance at 23 degrees in Figure $4 \mathrm{c}$. Furthermore, the equivalent radius of $r_{e q}^{\prime}$ (solid orange line) can be calculated by Equation S3.

Flow resistance of the elliptic channel. According to the flow resistance of Poiseuille's law in an elliptic channel, $Z=\frac{4 \eta L\left(r_{S D}^{2}+r_{U D}^{2}\right)}{\pi r_{S D}^{3} r_{U D}^{3}}$, we can analytically predict the tendency of flow resistance change, where $Z, L, \eta, r_{i}$ are the flow resistance, thickness of the deformed foil (equal to the length of nanopore), viscosity of water and the radius with $i$ indicating UD or SD. 


\section{Supplementary Figures and Tables}
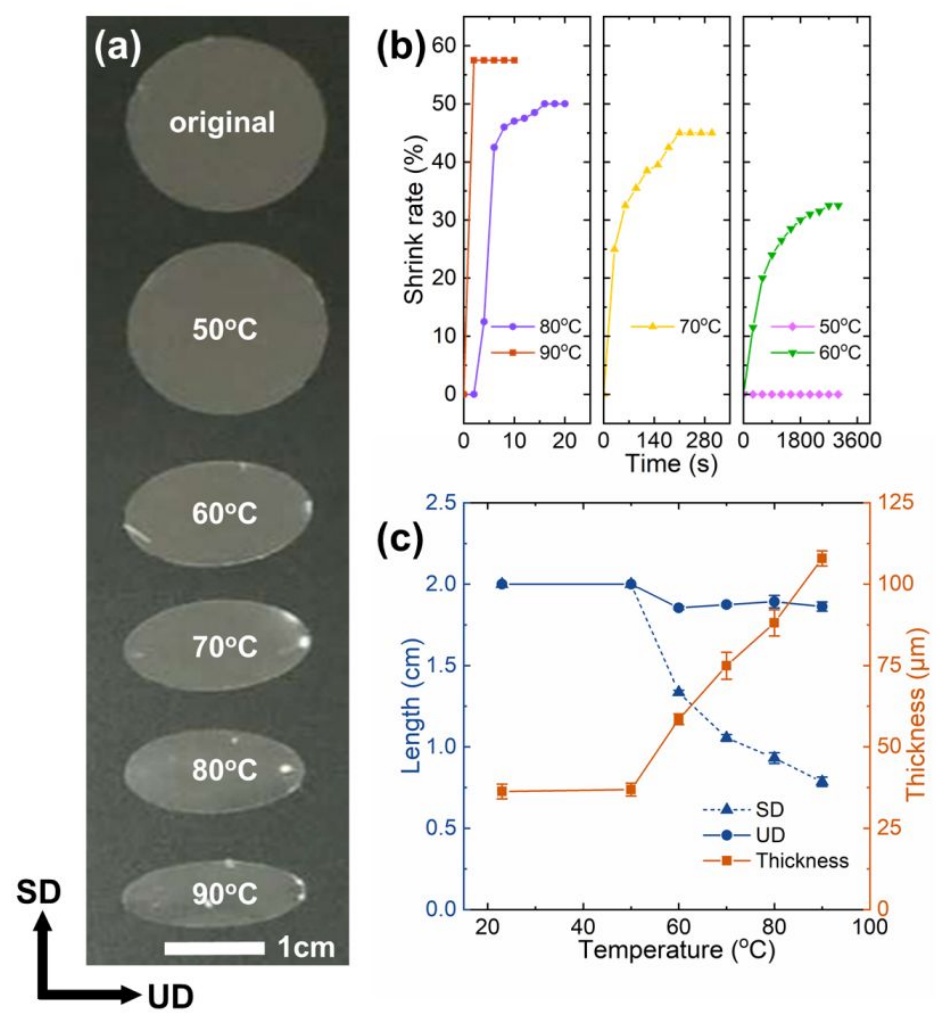

Figure S1. Shrinkage of oriented PET films at different temperatures. (a) Final saturated film after the thermal treatment of different temperatures. (b) The shrinking rate at SD as a function of the heating time. For instance, in a $60{ }^{\circ} \mathrm{C}$ water bath the foil started to shrink after 300 seconds, finally reaching a saturated value of 33\% shrinking rate after 45 minutes. In the $90{ }^{\circ} \mathrm{C}$ water bath, the shrinking rate becomes saturated within two seconds, with a maximum value of $58 \%$. (c) The equilibrium state of the film. We measured the length along the SD and UD axis and thickness of the foil after thermal treatment at different temperatures. The error bars are the standard deviation of five or more individual results. The steady-state length in the SD gradually decreases with rising temperature. The length in the UD on the other hand remains constant with a less than $10 \%$ change at all temperatures. The thickness of the polymer foil increases from $36.3 \mu \mathrm{m}$ to $107.9 \mu \mathrm{m}$ when treated at $90^{\circ} \mathrm{C}$, and we found that as a result the foil volume remains nearly constant while the area decreased (see Table S1). The results shown in Figure S1c demonstrated the 
shrinkage of polymer foil was temperature dependent, at least in the time range of our experiment, although long-term deformation in microscale was still possible ${ }^{3}$.

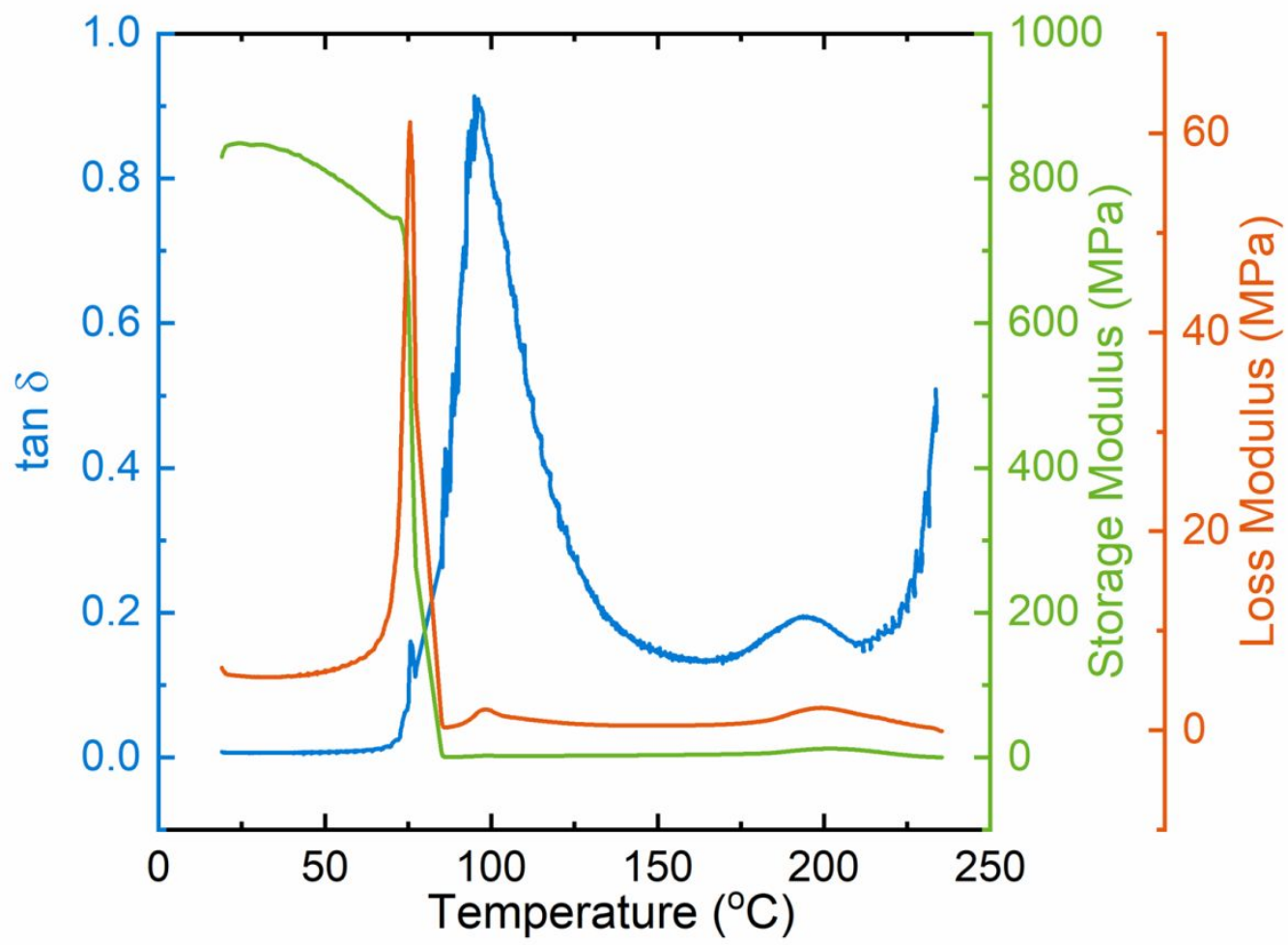

Figure S2. Dynamic thermomechanical analysis diagram of original PET films. The glass transition temperature of polymer showed the transition from an elastic state to glassy state. The peak of the loss factor $\tan \delta$ (blue) indicated the glass transition temperature is approximately $75^{\circ} \mathrm{C}$, where the loss factor is defined as the ratio of loss modulus to storage modulus. 


\section{(a)}

Nanopore fabrication

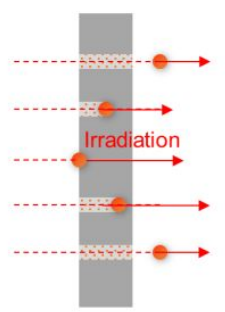

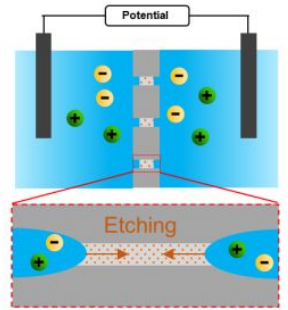

(b)

\section{Post processing}

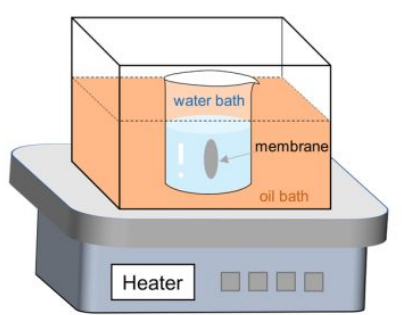

Figure S3. The fabrication process and post processing of the nanopores. (a) The fabrication process of ion track etched nanopores including irradiation and chemical etching. (b) Thermal treatment as the post processing. More details are shown in Methods.
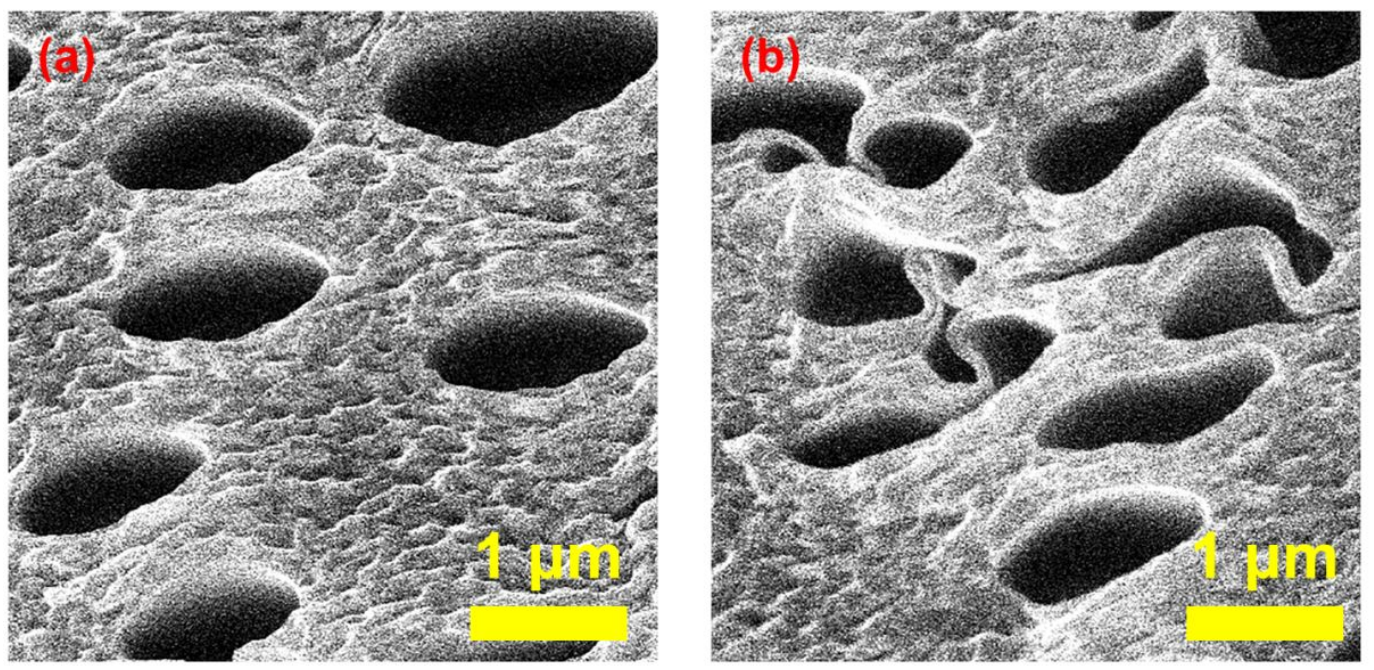

Figure S4. Unexpected deformation affected by neighbor pores. (a) showed the regular deformed pores which we counted. Due to the large distance between different pores, there is no obvious interaction between different pores, and each pore can be regarded as an isolated pore. The pores formed after the shrinkage can be regarded as the pores formed after shrinkage of a single pore. (b) Unexpected deformation induced by the interaction of neighbor pores. Because the distance between different pores is small, the interaction between different pores is more obvious, therefore, the shape after shrinking becomes irregular, and each pore will affect other pores in the area during the shrinking process. 

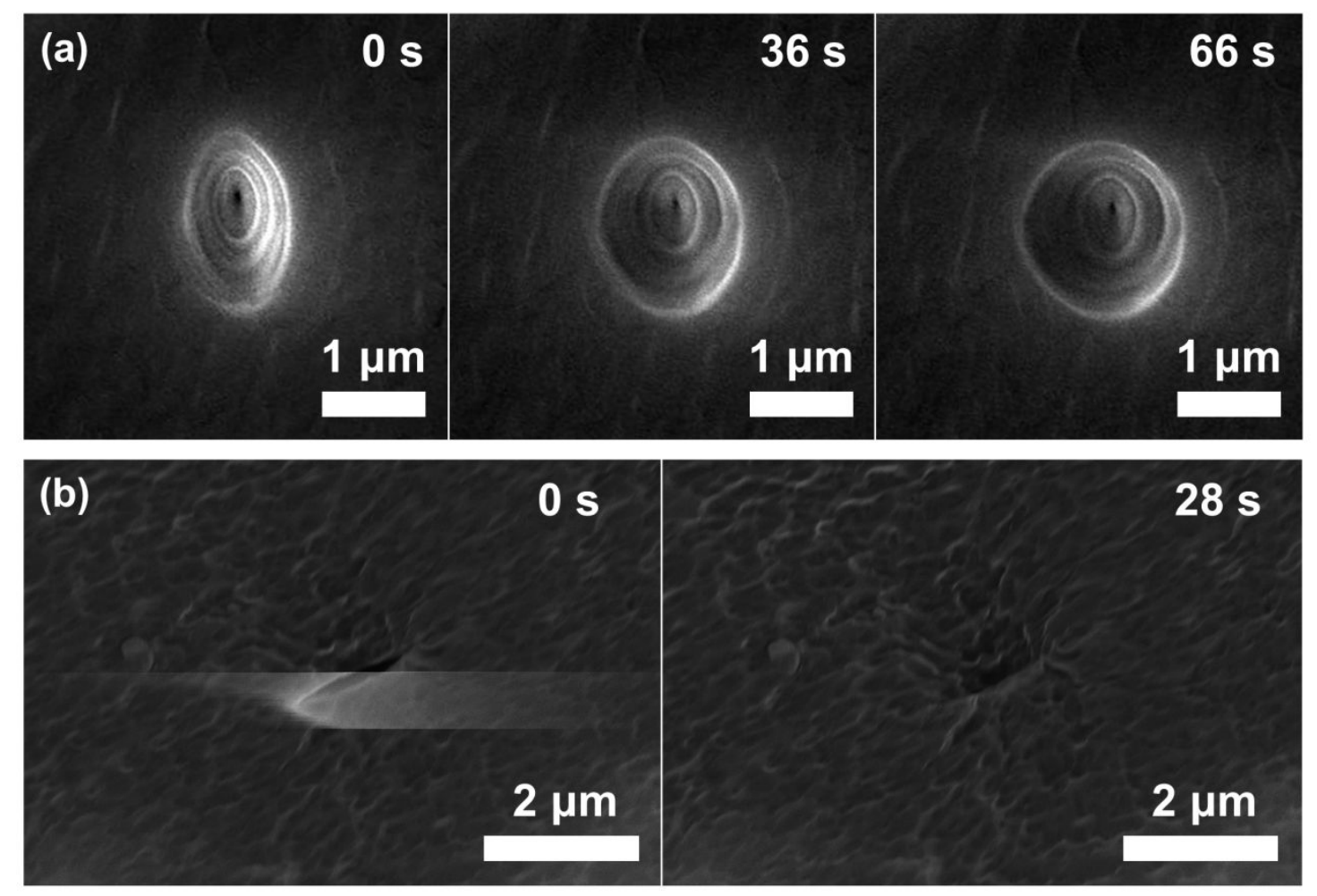

Figure S5. Pore deformation under electron beam irradiation. (a) The slightly elliptic nanopore turned into a circular nanopore after $66 \mathrm{~s}$ irradiation. (b) The slit after shrinkage gradually closed under irradiation. The polymer nanopore can deform under a certain time of electron beam irradiation $(15 \mathrm{kV}, 0.1 \mathrm{nA})$, which changed the topology of nanopores during the observation. As we described above, to avoid the deformation of nanopores by the electron beam irradiation, we make the imaging of nanopores as quick as possible. However, it is interesting to be noted that the deformations are relevant to the initial shape of the nanopores. For the nanopore before thermal treatment, the pore (slightly elliptic) turned to a perfect circular under electron beam irradiation, possibly caused by the edge tension as we showed. However, for a nanoslit after shrinkage, a certain time ( $\sim 2$ mins) of electron beam irradiation may induce complete close of the slit. We attributed this deformation to the thermal effects by electron beams irradiations, and the increase of temperatures enhanced the mobility of polymer molecules. Furthermore, during both nanopore deformations $r_{U D}$ remained constant during the e-beam induced deformation process, which makes our statistics convincing. However, for the "eye"-shaped nanopores, 
we didn't observe a clear change of the nanopore topology, which possibly indicated they were more thermally stable at high temperatures. 

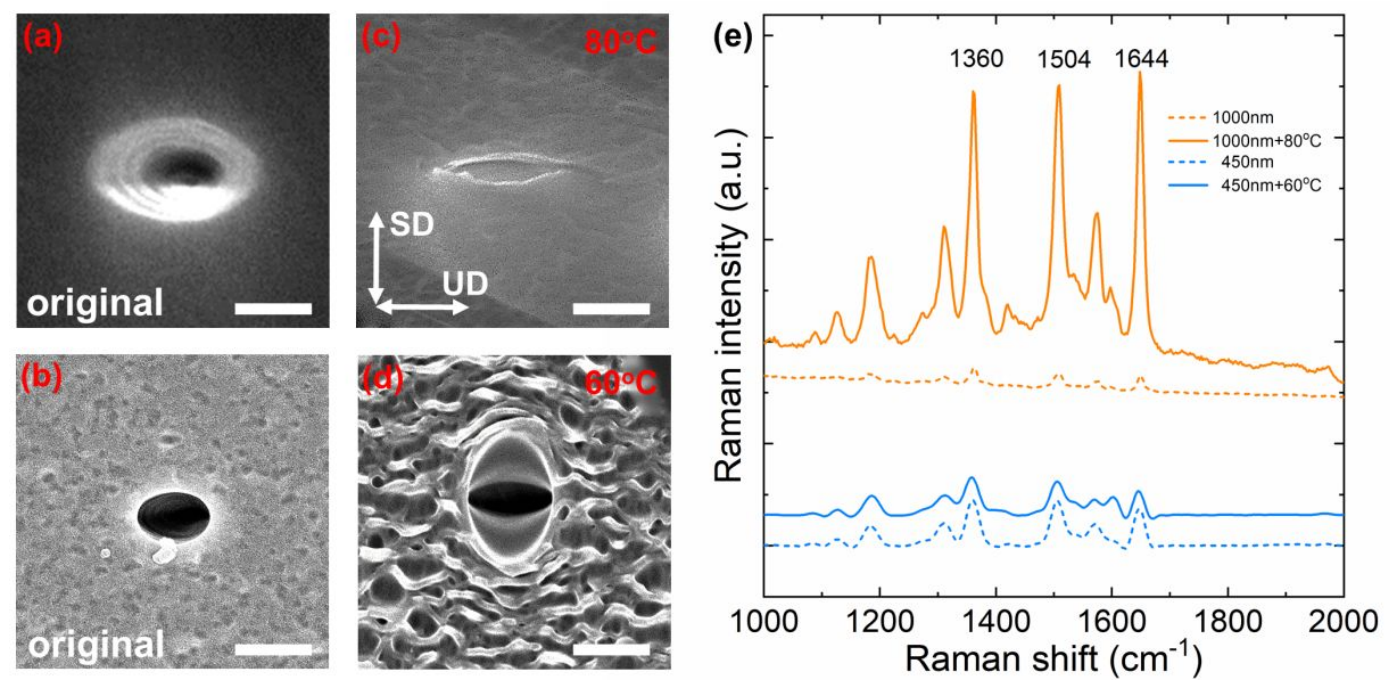

Figure S6. SERS of slit-like nanochannels after the thermal treatment. As the topology of nanopores transits to slit-like nanochannels, we suspect they could be used as the optical applications such as surface-enhanced Raman scattering (SERS), due to the downscaling of the nanopore in SD with the minimum width of $280 \mathrm{~nm}$ from SEM observations. Here we took a piece of multi-pore membrane to characterize the Raman signal enhancement after shrinkage. A thin layer $(15 \mathrm{~nm})$ of silver was evaporated on the surface of membrane (sputter PD300), and again use Rhodamine 6G solution $(0.1 \mu \mathrm{M})$ as analytes for the measurements.

The membrane was immersed in Rhodamine $6 \mathrm{G}$ solution for over 2 hours, ensuring the fully diffusion of Rhodamine molecules inside of the nanopores. We placed the samples under a Raman spectrometer (WITec, Alpha300R with working wavelength of $532 \mathrm{~nm}$ ) connected with a confocal microscope to measure the signals along the depth of a single nanopore. The results by using above four samples (Figure S6a-d) were shown in Figure S6e. We found a significant enhancement of Raman signals in all range above $750 \mathrm{~nm}$ for the deformed nanoslits. However, no clear difference was found for the nanopore of 450 $\mathrm{nm}$ after heating by $60^{\circ} \mathrm{C}$, possibly caused by the characterized areas exceeding the inner surface of the pore as expanding of the pore does not contribute to the signal enhancement. 

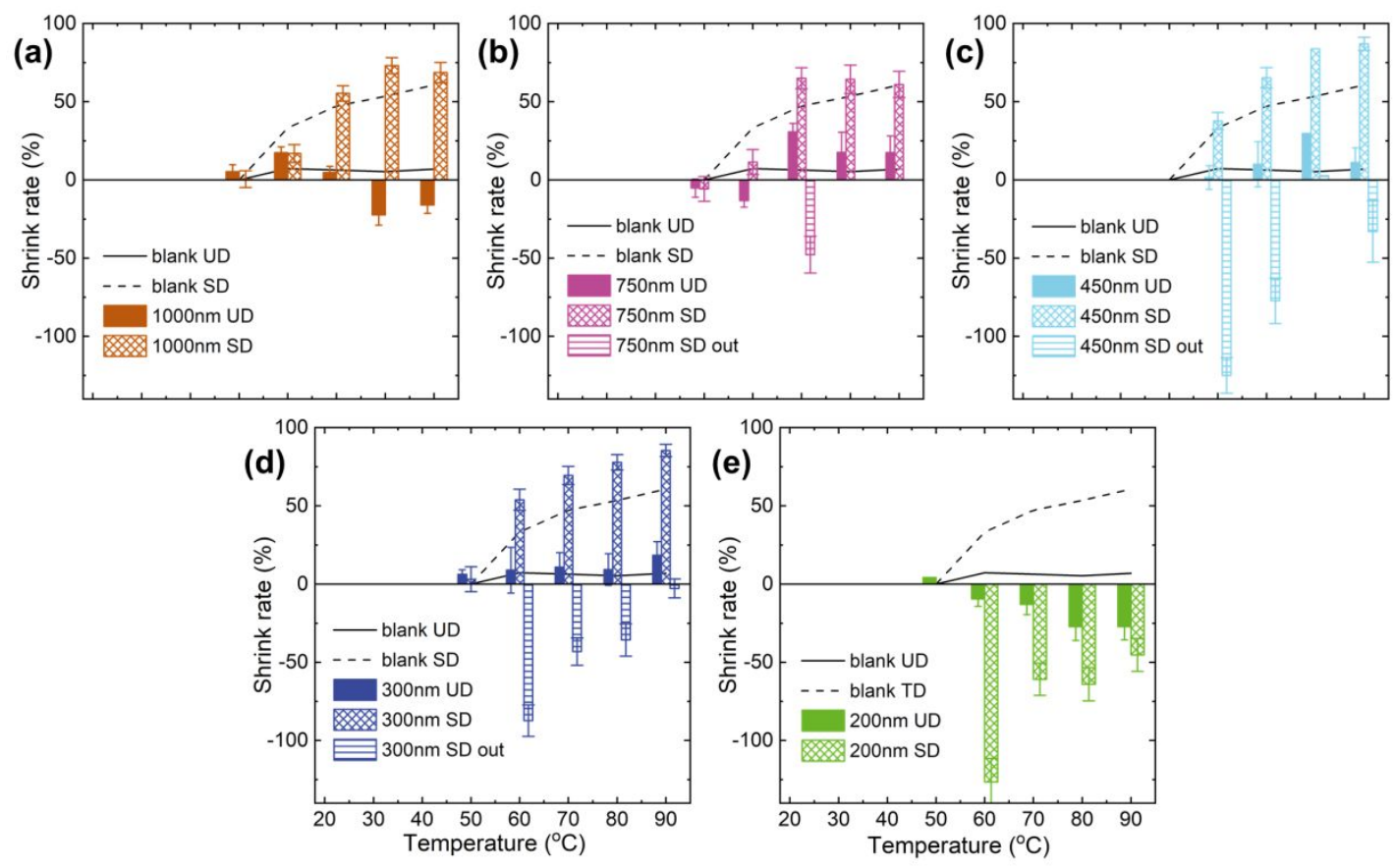

Figure S7. The statistical analysis of the pore deformation in SD and UD. Since observation of the nanopore deformation was not an in-situ experiment, we took the SD axis length to estimate the shrink rate, as we found polymer foil and nanopores in UD direction statistically remained nearly constant after the shrinkage process. We took the shrink rate of polymer foil into Figure S7 as a comparison, shown as dashed lines for SD and solid lines for UD. For the nanopores with radius of $\sim 200 \mathrm{~nm}$, the geometry remained circular. However, the pore size gradually increased resulting in a negative value of shrink rates controversial to the bulk polymer foil.

The deformations of nanopores were comparable when the pore radius range from 300 to $450 \mathrm{~nm}$. As the heating temperatures over $60^{\circ} \mathrm{C}$, the exterior surface slightly expanded shown as a negative value in Figure S7c and Figure S7d. The expansion of the exterior surface exposed the inner surface, which showed a comparable shrinkage to the polymer foil. The shrink rates of the nanopore inner surface were slightly higher than the bulk polymer foil (dashed lines), which may be overestimated as we use $l_{S D}$ before shrinkage to calibrate the shrink rate. The radius of the nanopore at the inner surface were indeed smaller than the exterior surface due to the conical shape caused by chemical etching. 
For the pore radius over $750 \mathrm{~nm}$, we didn't observe a two-layer shrinkage for most cases, which represented a similar shrink rate to the polymer foil that only existed in SD direction. However, the shrink rate of the nanopores in average is slightly larger than the bulk materials, possibly caused by the smaller elastic modulus due to the existence of cavities (nanopores) at the polymer surface ${ }^{4}$. 

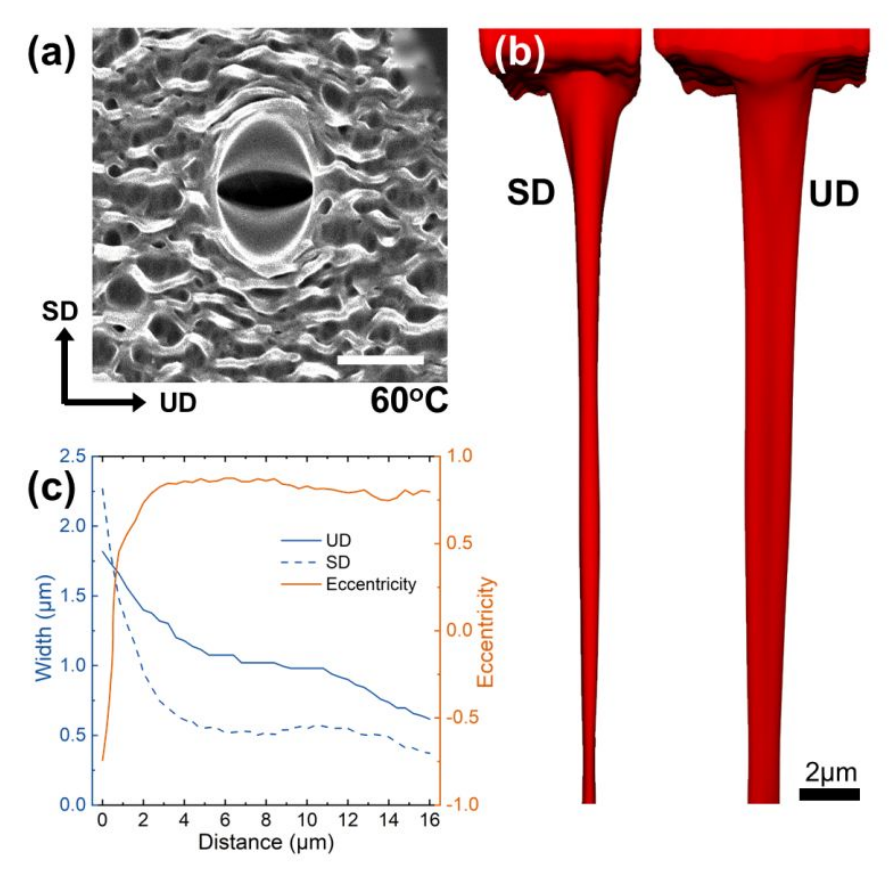

Figure S8. Characterization of the deformed nanopore by SEM and confocal microscopy. (a) The eye-shaped polymer nanopore after $60^{\circ} \mathrm{C}$ thermal treatment. The scale bar is $1 \mu \mathrm{m}$. (b) A confocal image of the nanopore shown in (a) across the polymer foil in the SD and UD. (c) The eccentricity of the nanopore in SEM shows an unequal deformation in the SD and UD at the outer and inner layers.

We filled fluorescent (Rhodamine B) solution in the nanopore and visualized the 3D pore morphology with a super-resolution confocal microscope (Leica TCS SP8 STED 3X). Hereby the filled area (nanopore) is imaged, leaving the geometry of the polymer nanopore empty as shown in Figure S8b. The images were filtered by deconvolution with Huygens Software for a better resolution. We took views in the SD and UD to illustrate the clearly different outlines of a single deformed nanopore in both directions.

In the UD view (right figure of Figure S8b), the nanopore has a smooth conical shape. This shape is caused by the chemical etching time being longer at the outer surface than at the inner surface, which is commonly found in ion track-etched nanopores. The angle of the conical shape in the UD is $3^{\circ}$, which is comparable to the classical ITE nanopore ${ }^{5}$.

We derived the nanopore extension in the two orthogonal directions SD (dashed line) 
and UD (solid line), shown in Figure S8c. The extension of the pore opening in the SD rapidly decreases until a depth of $\sim 4 \mu \mathrm{m}$ from the exterior surface of the polymer foil. The extension in the UD at the pore opening is smaller than the extension of the SD, but decreases less rapidly with depth. From the SD and UD extension we calculated the eccentricity $(e)$ of the nanopore as a function of the depth, by $e=\operatorname{sgn}\left(r_{U D}-r_{S D}\right)$ $\sqrt{\left|r_{U D}^{2}-r_{S D}^{2}\right|} / r_{U D}$, shown as the orange line in Figure S8c. The geometrical transition from (SD $>$ UD) to (UD $>$ SD) occurs at the depth of $0.5 \mu \mathrm{m}$ from the membrane surface as can be seen from the sign of eccentricity, after which point the geometry of the nanopore approaches the shrinkage of the polymer foil shown in Figure S8c. This unequal shrinkage of the nanopore in the SD and UD at the exterior surface and in the deeper layer of the foil causes the "eye"-shape of the nanopore. 


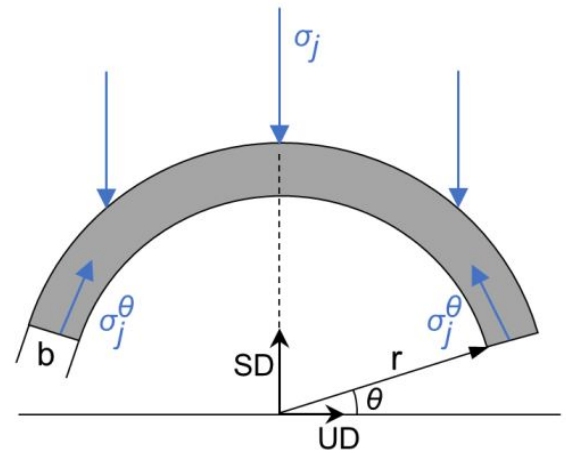

Figure S9. Hoop stress analysis of residual stress. For the oriented residual stress, due to neglectable radial stress, we calculate the hoop stress with force balance at $\mathrm{SD}, 2 \sigma_{j}^{\theta} b \cos \theta$ $=\sigma_{j} 2 r \cos \theta$ and we get $\sigma_{j}^{\theta}=\sigma_{j} r / b$, where the lower letter $j$ is in or out indicating inner or outer layer respectively. 


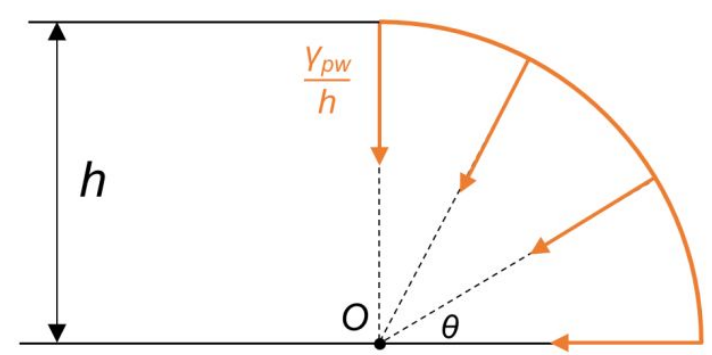

Figure S10. Polymer surface tension in water and horizontal component of edge tension. The surface tension of polymer film in water $\gamma_{p w}$ can be calculated ${ }^{6}$ by

$$
\gamma_{p w}=\gamma_{p}+\gamma_{w}-2 \Phi\left(\gamma_{p} \gamma_{w}\right)^{1 / 2}
$$

where $\gamma_{p}, \gamma_{w}$ and $\Phi$ are the surface tension of polymer films, the surface tension of water and a constant characteristic of the system. $\Phi$ can be approximately estimated ${ }^{6}$ by the expression

$$
\Phi=\frac{4\left(V_{p} V_{w}\right)^{1 / 3}}{\left(V_{p}^{1 / 3}+V_{w}^{1 / 3}\right)^{2}}=0.8923,
$$

where $V_{p}\left(139.13 \mathrm{~cm}^{3} / \mathrm{mol}\right)$ and $V_{w}\left(18 \mathrm{~cm}^{3} / \mathrm{mol}\right)$ are the molar volumes of PET polymer and water.

The surface tension of polymer film $\gamma_{p}$ is derived by

$$
\gamma_{p}=\gamma_{w}\left(\frac{(1+\cos \theta)^{2}}{4 \Phi^{2}}\right) .
$$

$\gamma_{w}$ is $71.8 \mathrm{mN} / \mathrm{m}$ at $23^{\circ} \mathrm{C}$ and the contact angle of water on the PET film we measured is $85^{\circ}$. Thus, we get $\gamma_{p}=26.7 \mathrm{mN} / \mathrm{m}$. Substitute $\gamma_{p}, \gamma_{w}$ and $\Phi$ into Equation $\mathrm{S} 5$, we can derive $\gamma_{p w}=20.4 \mathrm{mN} / \mathrm{m}$.

The curvature-induced edge tension $\gamma_{p w} / h$ is point to $O$ and we calculate the horizontal component of edge tension along the quadrant by 


$$
\sigma_{E d}=\frac{\int_{0}^{\pi / 2} \frac{\gamma_{p w}}{h} \cos ^{2} \theta h d \theta}{h}=\frac{\pi}{4} \frac{\gamma_{p w}}{h}
$$

where $\gamma_{p w}$ is the surface tension of polymer film in water. 


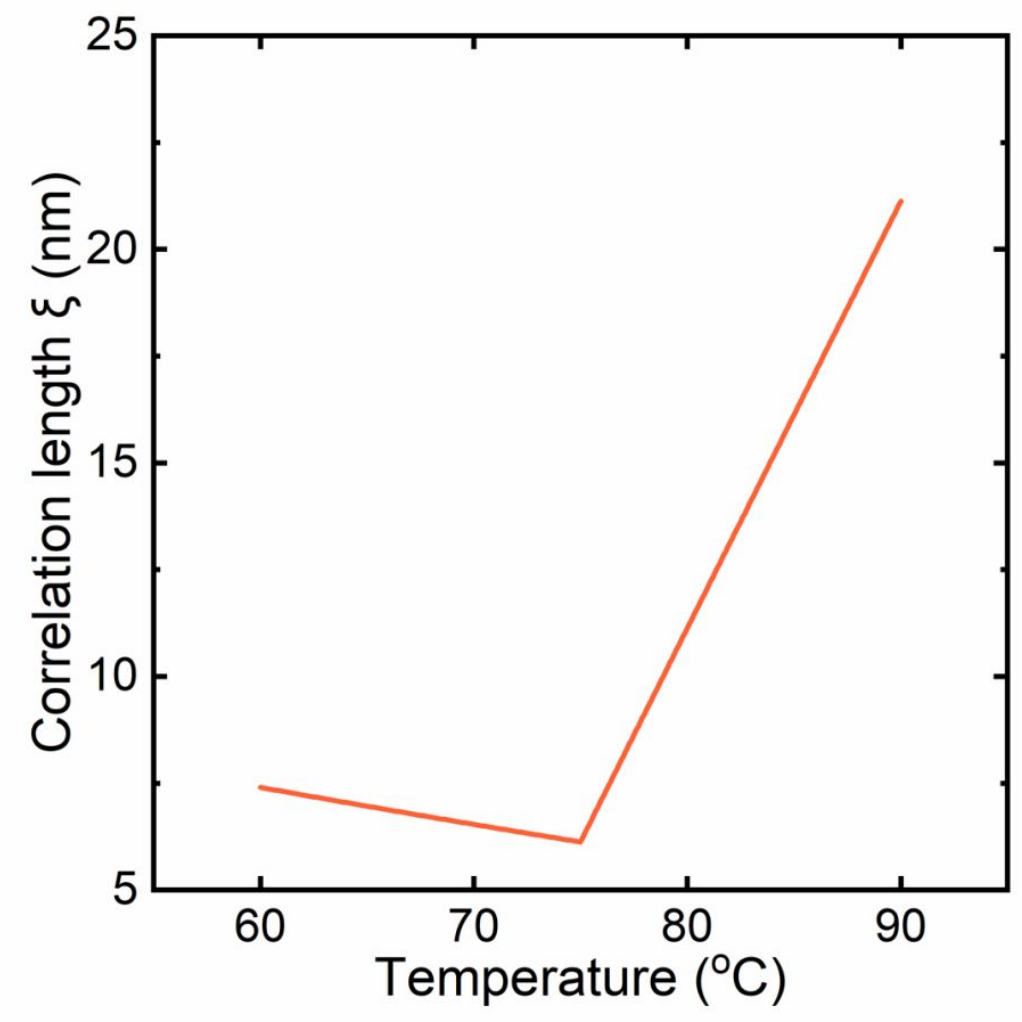

Figure S11. The correlation length as a function of temperatures. It has been proved that the surface layer thickness is obviously related to temperatures and declined below glass transition temperature ${ }^{7}$. The lower glass transition temperature of nanoporous polymer also demonstrate the existence of pores affects the thickness of surface layer ${ }^{8}$. The correlation length below glass transition temperature $\left(75^{\circ} \mathrm{C}\right)$ is given by $\xi=r_{0}+a\left(T_{\text {ons }}-T\right)^{b}$. Forrest and Mattsson ${ }^{7}$ took $r_{0}=6 \AA$ and got the fit values of $T_{\text {ons }}=485 \pm 6 \mathrm{~K}, b=2.00 \pm 0.1$ and $a=(2.95 \pm 0.3) \times 10^{-3}$. Above $75^{\circ} \mathrm{C}$, the correlation length will increase with temperatures 9,10 . Here we assume the correlation length increases linearly as a factor of $1 \mathrm{~nm} / \mathrm{K}^{10}$. 


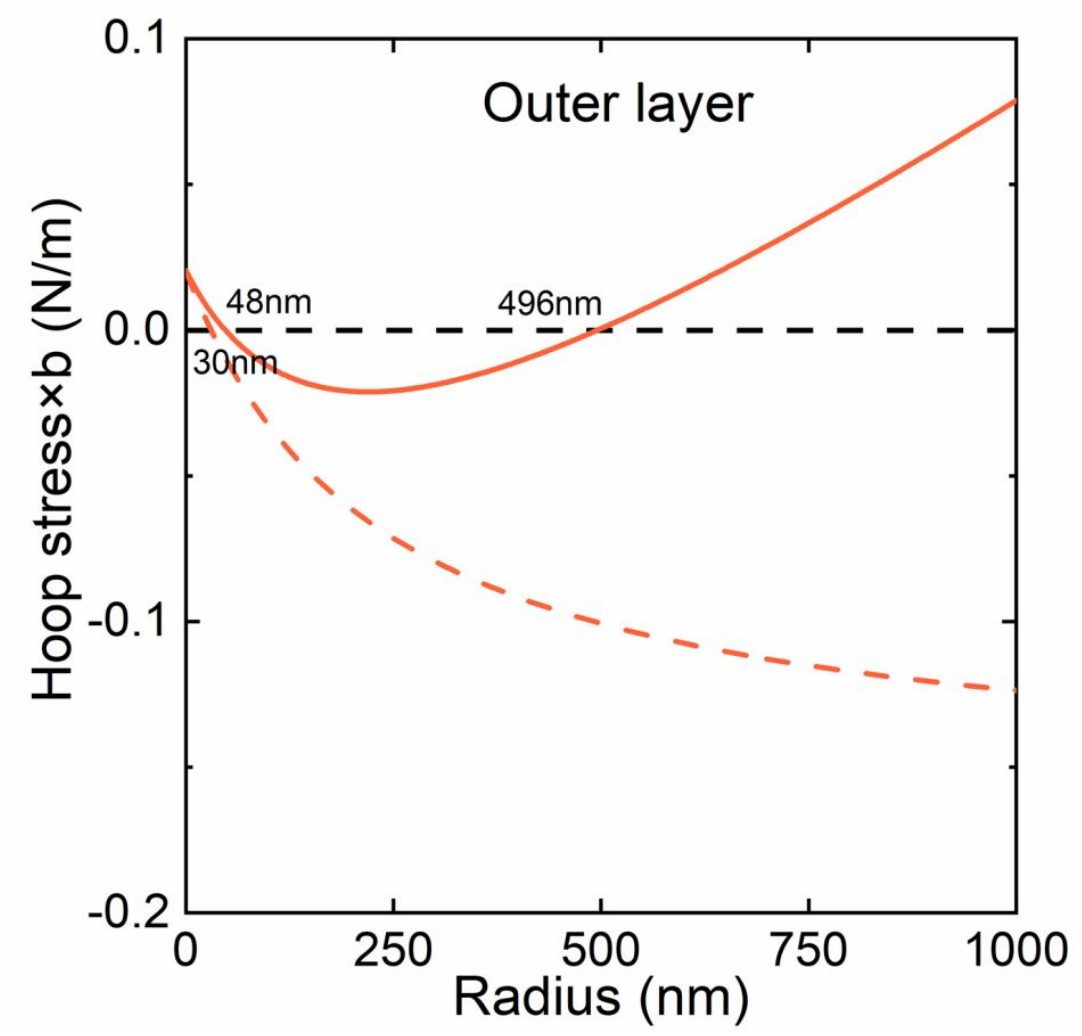

Figure S12. Two transition points at the outer layer. There is another transition point at 48 $\mathrm{nm}$ beyond our experiments opposite to the $496 \mathrm{~nm}$ from observation of experiments (solid line in Figure S12). This first transition point has been reported as the free energy competition ${ }^{11}$. The critical radius of this phenomena $r_{c}^{\#}$ can be describe with thickness of films $h^{\#}$ by

$$
r_{c}^{\#}=\frac{\pi}{4} h^{\#},
$$

We can calculate $h^{\#}=50.8 \mathrm{~nm}$ which is double $h(h=21.1+0.09 \mathrm{rmm})$ at $r=48 \mathrm{~nm}$, and $r_{c}^{\#}=39.9 \mathrm{~nm}$ which is smaller than critical radius of $48 \mathrm{~nm}$ in Figure $\mathrm{S} 12$. This may be from the addition of the residual stress. In comparison, the sum of Laplace pressure and edge tension except residual stress is plotted as the dotted line in Figure S12. The only transition point is located in $30 \mathrm{~nm}$ similar to the corresponding $r_{c}^{\#}=37.4 \mathrm{~nm}$ from Equation $\mathrm{S} 9$ which verified the assumption of surface layer thickness $h$. 
In summary, there might be two morphological transitions of nanopores in the thermal shrinkable film. Below first transition radius, the main mechanism is the competition of edge tension and Laplace pressure. However, the first critical radius is unobservable in our experiments. Above first transition radius, the residual stress began to have a significant effect which result in the second transition radius as found in our processes. 
(a)

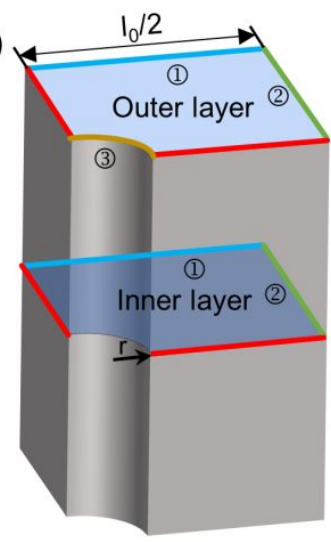

(b)

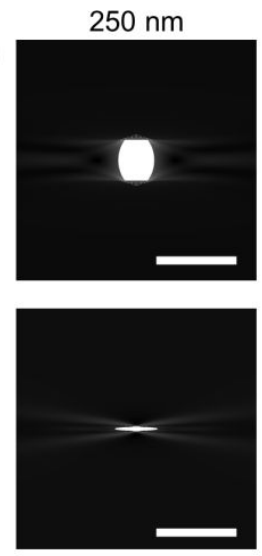

$450 \mathrm{~nm}$
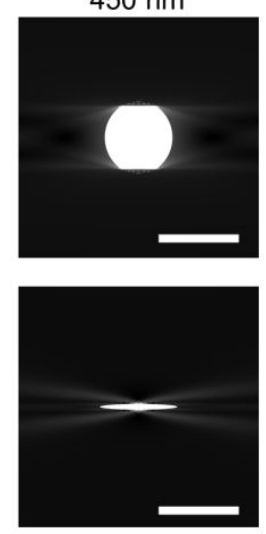

$1000 \mathrm{~nm}$

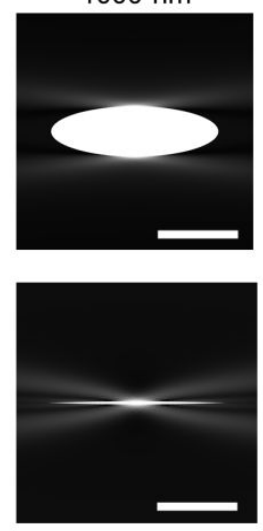

Figure S13. Simulation of deformation at the outer and inner layer of nanopore. (a) Scheme of simulation model. (b) Deformation of nanopores at the outer (top figures) and inner (bottom figures) layers with different radius of $250 \mathrm{~nm}, 450 \mathrm{~nm}$ and $1000 \mathrm{~nm}$. The scale bar is $1 \mu \mathrm{m}$. To understand the deformation of polymer nanopore after thermal shrinkage, hereby we use a linear elastic model to illustrate the deformed topologies by using COMSOL 5.3. We set the thermal shrinkage stress of polymer nearby nanopore to be same as the bulk polymer foil, with $2.5 \%$ of stress was used at the outer surface. We set a quarter of the circular PET nanopore before shrinkage, with a symmetrical boundary at the side of the pores shown as red lines, where $l_{0}=12 \mu \mathrm{m}$ is the length of the material for isolated single pore, $r$ is the radius of the pore. Because the small deformation in UD of polymer foil, so we assume the modulus of UD is much greater than SD. At the outer and inner layer, the shrinkage forces 0.2 $\mathrm{MPa}$ and 8.1 MPa are applied at the blue (1) sides of inner and outer layer respectively, and no force at the green (2) sides. Besides, one expansion force is increased at the orange (3) side in the outer layer, as shown in the Figure S13a. The density of foil is $1380 \mathrm{~kg} / \mathrm{m}^{3}$. We set modulus $E_{U D}=1000 \mathrm{MPa}, E_{S D}=13.5 \mathrm{MPa}$ for the inner layer, and the modulus of the outer layer is $2.5 \%$ of the inner layer.

At the outer layer, we find when the expansion force is equal to $1 \mathrm{MPa}, 0.75 \mathrm{MPa}$ and 0.2 $\mathrm{MPa}$ for different radius of $250 \mathrm{~nm}, 450 \mathrm{~nm}$ and $1000 \mathrm{~nm}$, the simulation results fit well with our experiments (the top pictures in Figure S13b). The less shrinkage force at the outer layer of polymer film induced the asymmetric deformation of nanopore at exterior and inner surfaces of the nanopore. The small deformation in UD direction is probably caused by the large modulus of UD, matched well with the experimental characterizations. 
However, the inner layer of nanopore (the bottom pictures in Figure S13b) remains shrinkage for all range of pore size, due to lack of the expansion force. It demonstrated that the reduction of shrink forces at the outer layer is pore size dependent, causing the different topology of deformed nanopore after shrinkage. 

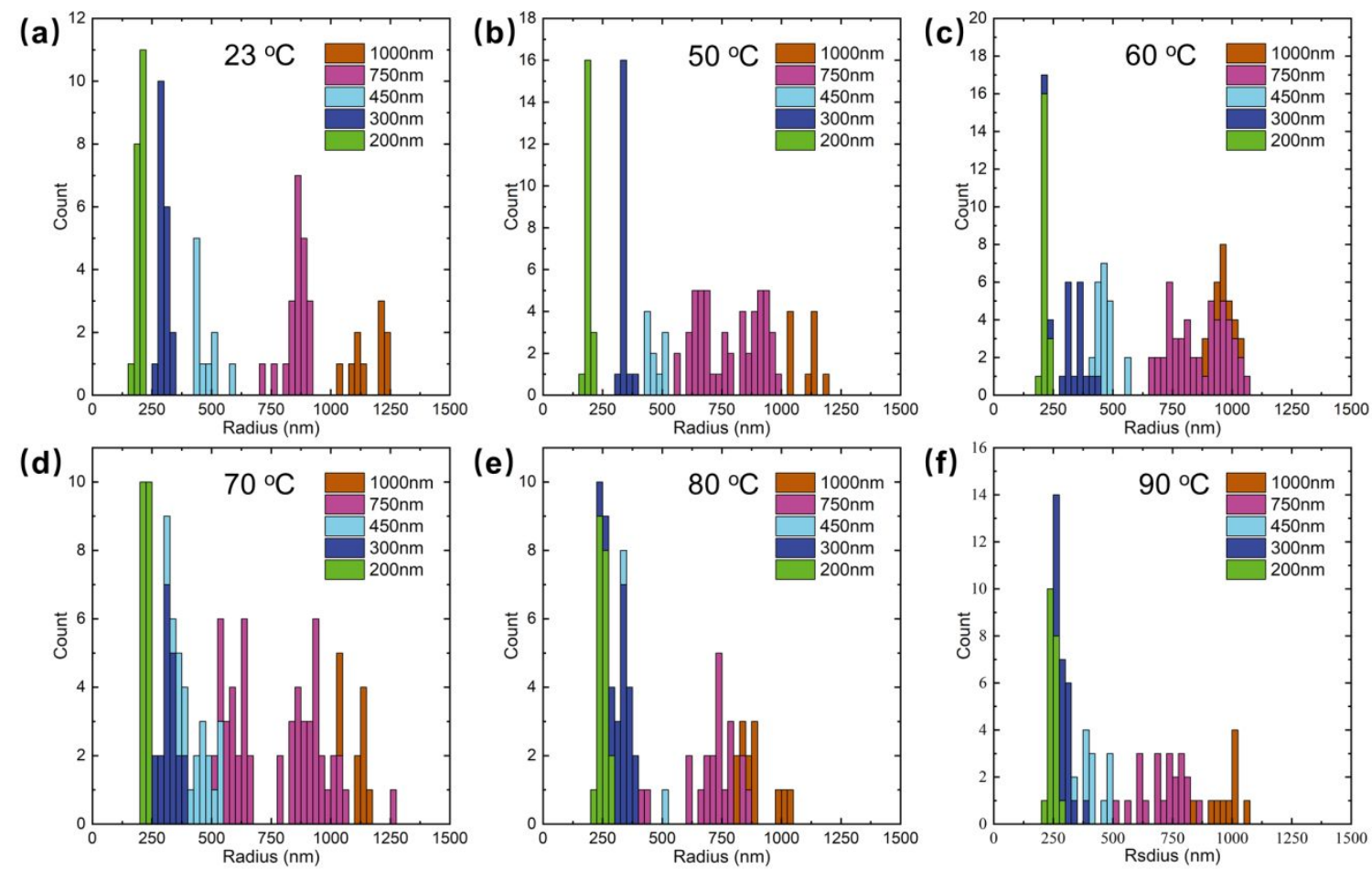

Figure S14. Distribution of pore radius at various temperatures. We statistically counted the pore radius in UD direction as a function of temperatures of 23, 50, 60, 70, 80 and 90 ${ }^{\circ} \mathrm{C}$ with an interval of $25 \mathrm{~nm}$. In the phase diagram (Figure 3d), pores of $200 \mathrm{~nm}$ mainly deformed into circular nanopores, pores of $300 \mathrm{~nm}$ and $450 \mathrm{~nm}$ mainly deformed into "eye"-shaped nanopores and pores of $750 \mathrm{~nm}$ and $1000 \mathrm{~nm}$ mainly deformed into slits. But there are some areas where the transition between two states occurs. Different pore sizes (colors) separated from each other at 23 and $50{ }^{\circ} \mathrm{C}$, but overlapped over $60{ }^{\circ} \mathrm{C}$. In the overlap area of $200 \mathrm{~nm}$ and $300 \mathrm{~nm}$, one transition from circular pores to apparent "eye"shaped pores occurs. In the overlap area of $450 \mathrm{~nm}$ and $750 \mathrm{~nm}$, another transition from apparent "eye"-shaped pores to nanoslits occurs. 

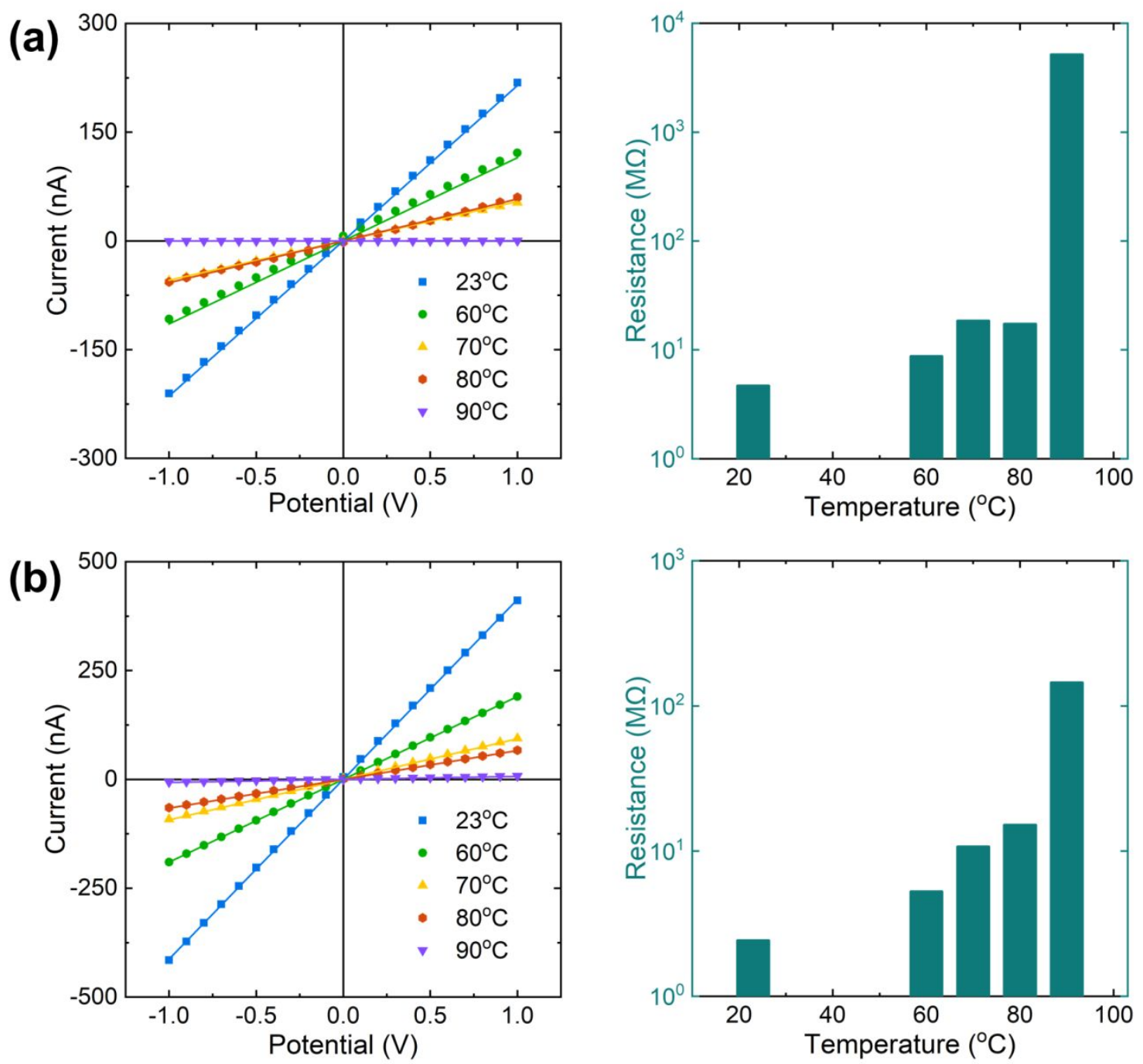

Figure S15. Temperature modulated ionic transport through two films containing single nanopore. The maximum reduction can reach $99.91 \%$ for (a) and $98.33 \%$ for (b). 

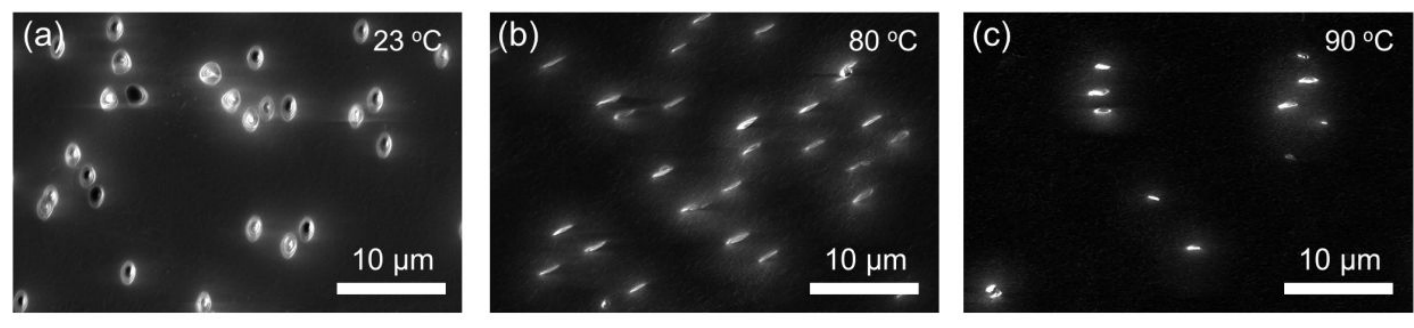

Figure S16. The change of pore density after various thermal treatment. (a) Morphology of $1 \mu \mathrm{m}$ radius pores at $23{ }^{\circ} \mathrm{C}$. (b) Morphology of $1 \mu \mathrm{m}$ radius pores at a shrinkage temperature of $80^{\circ} \mathrm{C}$. (c) SEM image of $1 \mu \mathrm{m}$ radius pores at a shrinkage temperature of $90{ }^{\circ} \mathrm{C}$, which showed a clear reduction of nanopore density. The fully close of partial nanopores probably results in a significant increase of flow resistance after $90{ }^{\circ} \mathrm{C}$ heating. 
Table S1. Volume change of film versus temperatures

\begin{tabular}{c|c|c|c|c|c|c}
\hline Temperature $\left({ }^{\circ} \mathrm{C}\right)$ & 23 & 50 & 60 & 70 & 80 & 90 \\
\hline $\mathrm{SD}(\mathrm{mm})$ & 20 & 20 & 13.4 & 10.6 & 9.3 & 7.9 \\
\hline $\mathrm{UD}(\mathrm{mm})$ & 20 & 20 & 18.5 & 18.7 & 18.9 & 18.6 \\
\hline Thickness $(\mathrm{mm})$ & 0.0363 & 0.0369 & 0.0585 & 0.0749 & 0.0881 & 0.1079 \\
\hline Volume $\left(\mathrm{mm}^{3}\right)$ & 11.40 & 11.59 & 11.39 & 11.66 & 12.16 & 12.45 \\
\hline
\end{tabular}

This table summarized the lengths of polymer films in SD and UD directions and the thickness change under different temperatures. Our results indicated that the volume of the film slightly increases less than $10 \%$. The slight increase of volume was possibly caused by the increase of stiffness after heating, which resulted in a smaller strain by clamping the films during measurement. 


\section{References}

(1) Long, S. D.; Ward, I. M. Shrinkage Force Studies of Oriented Polyethylene Terephthalate. J. Appl. Polym. Sci. 1991, 42, 1921-1929.

(2) Pinnock, P. R.; Ward, I. M. Stress-Optical Properties of Amorphous Polyethylene Terephthalate Fibres. Trans. Faraday Soc. 1966, 62, 1308-1320.

(3) Chai, Y.; Salez, T.; McGraw, J. D.; Benzaquen, M.; Dalnoki-Veress, K.; Raphaël, E.; Forrest, J. A. A Direct Quantitative Measure of Surface Mobility in a Glassy Polymer. Science 2014, 343, 994-999.

(4) Herrmann, M.; Schwarzer, N.; Richter, F.; Frühauf, S.; Schulz, S. E. Determination of Young's modulus and yield stress of porous low-k materials by nanoindentation. Surf. Coat. Technol. 2006, 201, 4305-4310.

(5) Apel, P. Y.; Korchev, Y. E.; Siwy, Z.; Spohr, R.; Yoshida, M. Diode-like singleion track membrane prepared by electro-stopping. Nucl. Instrum. Methods Phys. Res. B 2001, 184, 337-346.

(6) Girifalco, L. A.; Good, R. J. A Theory for the Estimation of Surface and Interfacial Energies .1. Derivation and Application to Interfacial Tension. J. Phys. Chem. 1957, 61, 904-909.

(7) Forrest, J. A.; Mattsson, J. Reductions of the glass transition temperature in thin polymer films: Probing the length scale of cooperative dynamics. Phys. Rev. E 2000, 61, R53-R56.

(8) Liu, T.; Ozisik, R.; Siegel, R. W. Pore structure and glass transition temperature of nanoporous poly(ether imide). J. Polym. Sci., Part B: Polym. Phys. 2006, 44, 3546-3552. 
(9) Wei, S. A.; Gallino, I.; Busch, R.; Angell, C. A. Glass transition with decreasing correlation length during cooling of Fe50Co50 superlattice and strong liquids. Nat. Phys. 2011, 7, 178-182.

(10) Ceresoli, M.; Volpe, F. G.; Seguini, G.; Antonioli, D.; Gianotti, V.; Sparnacci, K.; Laus, M.; Perego, M. Scaling of correlation length in lamellae forming PS-b-PMMA thin films upon high temperature rapid thermal treatments. J. Mater. Chem. C 2015, 3, 86188624.

(11) Ilton, M.; DiMaria, C.; Dalnoki-Veress, K. Direct Measurement of the Critical Pore Size in a Model Membrane. Phys. Rev. Lett. 2016, 117, 257801. 